\title{
Performance of Cladding-Pumped Few-Mode EDFAs in Optical Space-Division Multiplexed Systems
}

\author{
Hesham A. Youssef ${ }^{\star, \#}$, and Ziad A. El-Sahn ${ }^{\S}$ \\ ${ }^{\star}$ IT Department, Institute of Graduate Studies and Research, Alexandria University, Alexandria 21526, Egypt \\ "International Transmission Sector, Telecom Egypt, Alexandria, Egypt. Email: hesham.yousef@te.eg \\ ${ }^{\S}$ Photonics Group, Electrical Engineering Department, Alexandria University, Alexandria 21544, Egypt
}

\begin{abstract}
We report a cladding-pumped 10-modes EDFA with modal gain $>20 \mathrm{~dB}$ over the $\mathrm{C}$-band, zero differential modal gain and $\rightarrow 9$ dB noise figure. Further, we successfully demonstrate a BER below $10^{-10}$ at $400 \mathrm{~Gb} / \mathrm{s}$.
\end{abstract}

\section{INTRODUCTION}

With the information carrying capacity of single-mode fiber (SMF) close to fundamental limits, there is an interest in space-division multiplexing (SDM) to increase the system capacity [1]. Mode-division multiplexing (MDM) transmission over few-mode fibers (FMFs) is a promising candidate for SDM systems [1]-[3]. However, the design of energy efficient optical amplifiers for SDM long haul systems is very crucial. To date, few-mode erbium doped fiber amplifiers (FM-EDFAs) that simultaneously amplify up to 4 linearly polarized (LP) mode groups (i.e. 6-spatial modes) have been experimentally demonstrated and a differential modal gain (DMG) of less than $2 \mathrm{~dB}$ has been achieved using a ring-doped erbium doped fiber (EDF) combined with bidirectional higher-order mode $\left(\mathrm{LP}_{21}\right)$ pumping [4].

Recently, we demonstrated via simulations a FM-EDFA supporting up to 10 -spatial modes with zero DMG, and a bit error rate below $10^{-10}$ at $10 \mathrm{~Gb} / \mathrm{s}$ per mode by using an optimized ring-doped EDF and bi-directional $\mathrm{LP}_{21}$ pumping scheme [5]. However, scaling up the modes beyond 10 makes the design more challenging as it will require higher pump power levels. Moreover, multiplexing several single-mode pumps to generate sufficient pump power is an expensive way of pumping such amplifiers, even if it is technically possible. Cladding pumping represents a promising that addresses these issues [6]. High power (up to $10 \mathrm{~W}$ ), low cost multimode pump diodes operating in the multi-watt regime are now readily available given the emergence and commercial success of the high power cladding pumped fiber laser. Recently, a FM-EDFA supporting 6-spatial modes with a cladding pumped architecture has been experimentally demonstrated; a signal gain of $>20 \mathrm{~dB}$ was reported for all the guided modes with a DMG of $\sim 3 \mathrm{~dB}$ and noise figure (NF) of 6-7 $\mathrm{dB}$ [6].

In this paper, we extend the idea of a cladding pumped FMEDFA beyond 6-spatial modes. We first validate the results of 6-spatial modes via simulations. Herein, we present results for the case of 10-spatial modes in an SDM system to achieve high gain while maintaining a low DMG. Achieving an accepted NF is also one of the main objectives. We also test the BER performance of the system with the proposed FMEDFA at high data rate $(40 \mathrm{~Gb} / \mathrm{s})$ per mode and up to 12 spatial modes.

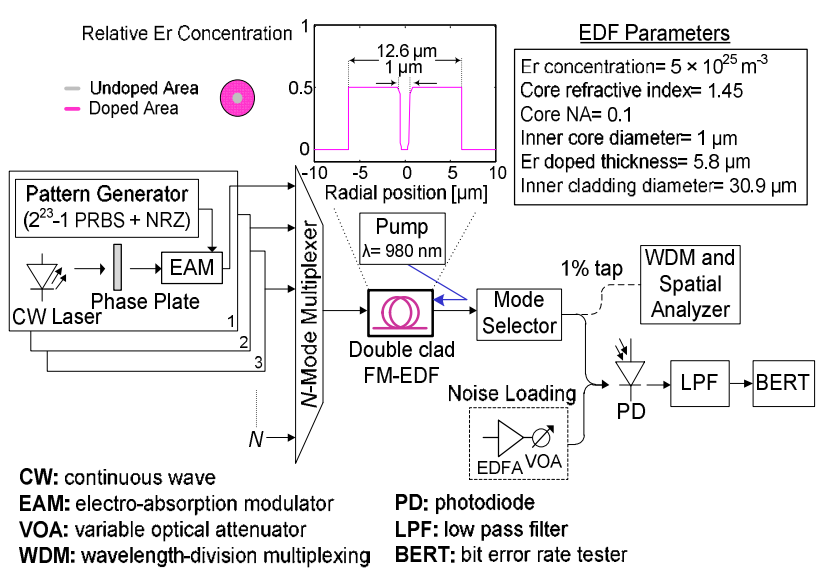

Fig. 1. Proposed cladding pumped FM-EDFA in an IM/DD SDM system over a back-to-back configuration.

\section{SYSTEM ARCHITECTURE OF AN SDM SYSTEM WITH A Cladding PuMPED FM-EDFA}

Fig. 1 shows a generic intensity modulation/direct detected (IM/DD) SDM system with the proposed cladding pumped FM-EDFA over a back-to-back configuration. Phase plates are used at the transmitter after the laser diodes to selectively excite the desired signal modes. A $40 \mathrm{~Gb} / \mathrm{s} 2^{23}-1$ pseudo random binary sequence (PRBS) non-return-to-zero (NRZ) signal from a pattern generator is applied to an electroabsorption modulator (EAM). An $N$-mode multiplexer (NMMux) (ideal power combiner used for emulation) then combines the signals from different transmitters. The output signal modes from the NM-Mux is then launched to a double clad few-mode erbium doped fiber (FM-EDF), in which the erbium ions are substantially confined within the ring inside the fiber core to help mitigate the DMG as per inset. The double clad fiber is designed with the parametres listed in Fig. 1. The fiber is pumped with a $980 \mathrm{~nm}$ counter-directional multimode pump. At the receiver side, a mode selector is used to select the signal mode under test. Wavelength-division multiplexing (WDM) and spatial analyzers are used to display the output signal mode and determine the output signal and noise power, and hence the gain and NF of individual channels. A conventional EDFA followed by a variable attenuator is added at the receiver to control the optical signalto-noise ratio (OSNR) for the BER calculations. After photodetection, a Gaussian low pass filter (LPF) is used to remove out-of-band noise. A bit error rate tester (BERT) then estimates the BER for each mode signal separately. 

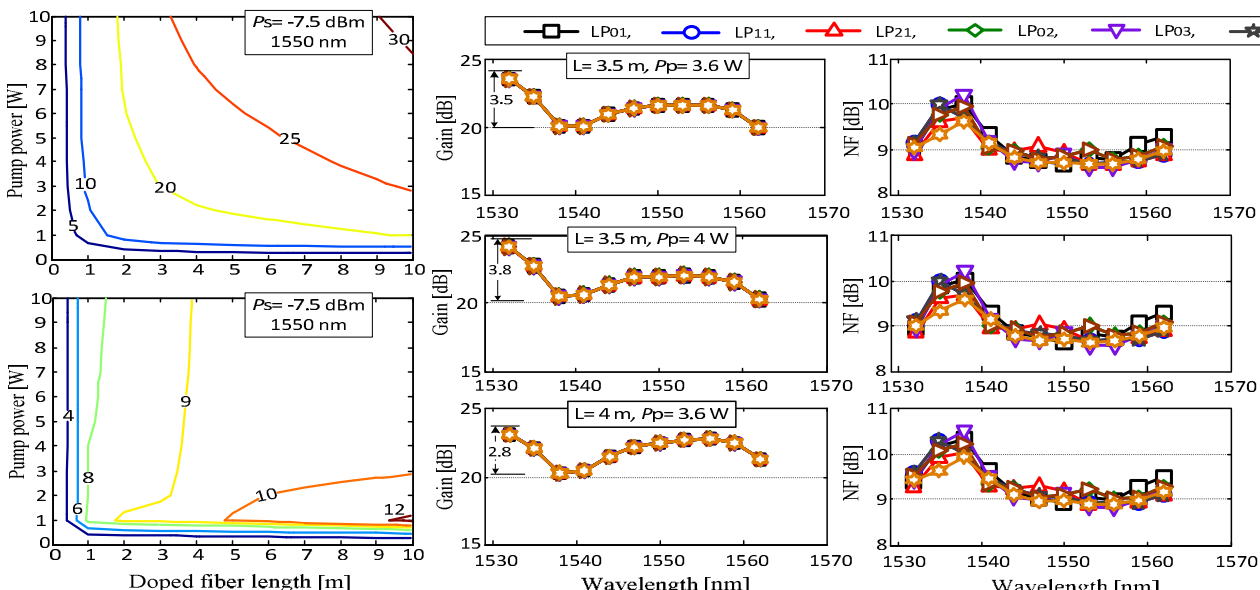

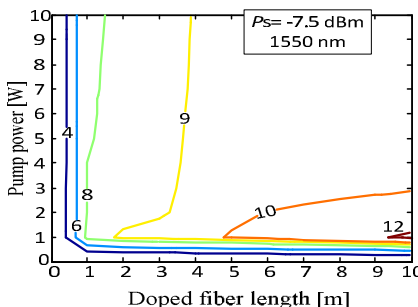

(a) (b)

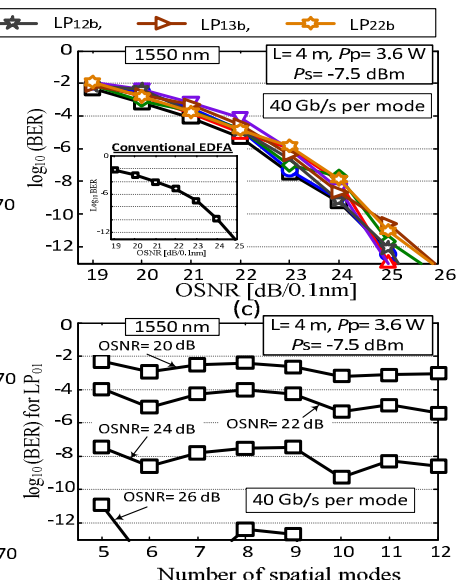

(d)

Fig. 2. (a) Gain (top) and average noise figure (bottom) contours as a function of doped fiber length and pump power $(P p)$, (b) gain spectra (left) and noise figure (right) for all the tested modes at different EDF lengths and pump powers $(P \mathrm{p}),(\mathrm{c}) \mathrm{BER}$ as a function of OSNR for the 10M-EDFA, and $(\mathrm{d}) \mathrm{BER}$ for LP $\mathrm{P}_{01}$ versus number of spatial modes at different OSNR levels. The input signal power $(P \mathrm{~s})$ is $-7.5 \mathrm{dBm}$ per mode.

\section{PERFormance of THE PRoposed FM-EDFA}

We test the gain, NF and BER performance of the FMEDFA supporting 10-spatial modes with the 8-spatial mode groups ( $\mathrm{LP}_{01}, \mathrm{LP}_{11}, \mathrm{LP}_{21}, \mathrm{LP}_{02}, \mathrm{LP}_{03}, \mathrm{LP}_{12 \mathrm{~b}}, \mathrm{LP}_{13 \mathrm{~b}}$, and $\mathrm{LP}_{22 \mathrm{~b}}$ ). A $1550 \mathrm{~nm}$ signal is chosen for the different modes and with input signal power of $-7.5 \mathrm{dBm}$ per mode. Fig. 2a (top) shows contour plot of the FM-EDFA gain as a function of EDF length and pump power $(P \mathrm{p})$, where the DMG is equal to zero at all points. Due to insufficient pump power, a small gain of $<20 \mathrm{~dB}$ is observed at the EDF length and pump power of 0-3 $\mathrm{m}$ and 0-3 W, respectively. The gain increases with an increase in either the doped fiber length or the pump power. The average NF contour plot of the tested modes is also shown in Fig. $2 \mathrm{a}$ (bottom). For the pump power of 0-3 W, NF increases from $4 \mathrm{~dB}$ to $12 \mathrm{~dB}$ with an increase in the EDF length from $0.5 \mathrm{~m}$ to $10 \mathrm{~m}$. That is mainly due to insufficient population inversion due to the limitation in the allowable pump power. However, at the pump power of $>3 \mathrm{~W}$, NF remains in the region of $9-10 \mathrm{~dB}$ with increasing the EDF length to $3.5-10 \mathrm{~m}$.

Three points are chosen from Fig. 2a (top), at which the gain of the $1550 \mathrm{~nm}$ mode signal is $>20 \mathrm{~dB}$ to evaluate the gain performance of the 10M-EDFA under a wavelengthdivision multiplexing (WDM) configuration across the C-band $(1532-1562 \mathrm{~nm})$. The evaluated gain and NF across the Cband at EDF length of $3.5 \mathrm{~m}$ and pump power of $3.6 \mathrm{~W}$ is illustrated in Fig. 2b (top). As shown, the gain is $\geq 20 \mathrm{~dB}$ for all the tested modes with zero DMG controlled over the Cband and gain flatness of $3.5 \mathrm{~dB}$. The $\mathrm{NF}$ is $\sim 9 \mathrm{~dB}$ for wavelengths longer than $1540 \mathrm{~nm}$ as shown in Fig. 2b (top). The same is observed in case of same EDF length and pump power of $4 \mathrm{~W}$, but with a bit higher modal gain $(>20 \mathrm{~dB})$ and gain flatness of $3.8 \mathrm{~dB}$ as shown in Fig. $2 \mathrm{~b}$ (middle). A modal gain of $>20 \mathrm{~dB}$ with optimum gain flatness of $2.8 \mathrm{~dB}$ is observed for all the tested modes at EDF length of $4 \mathrm{~m}$ and pump power of $3.6 \mathrm{~W}$ as shown in Fig. $2 \mathrm{~b}$ (bottom) and the estimated NF is $\sim 9 \mathrm{~dB}$ with a standard deviation of $\pm 0.08 \mathrm{~dB}$ at $1550 \mathrm{~nm}$.
We evaluate the BER performance of the system for the different numbers of modes and up to 12-spatial modes at the optimum point of $4 \mathrm{~m}$ EDF length and $3.6 \mathrm{~W}$ pump power. Fig. 2c shows the BER versus the OSNR for the 10-spatial modes at $40 \mathrm{~Gb} / \mathrm{s}$ per mode signal. As shown, BER below $10^{-10}$ is achieved for all the tested modes at an OSNR of $25 \mathrm{~dB}$ with a penalty of around $6 \mathrm{~dB}$ compared with our previous core pumping results in case of $10 \mathrm{~Gb} / \mathrm{s}$ signal per mode [5]. It can be noticed that all channels behave similarly, which confirms the zero DMG and the same NF in Fig. 2b. The same BER is observed for a conventional single-mode EDFA with $20 \mathrm{~dB}$ gain and NF of $4 \mathrm{~dB}$ as per inset. Furthermore, the BER for $\mathrm{LP}_{01}$ signal mode is calculated for different number of modes up to 12-spatial modes at different OSNR levels. The BER is below $10^{-10}$ for the different number of modes at an OSNR of $26 \mathrm{~dB}$ as shown in Fig. $2 \mathrm{~d}$.

\section{CONCLUSION}

We evaluated for the first time to our knowledge an SDM system performance with a cladding pumped FM-EDFA supporting 10-spatial modes. A modal gain of $>20 \mathrm{~dB}$ across the $\mathrm{C}$-band is reported for all the tested modes with zero DMG and gain flatness of $<3 \mathrm{~dB}$ and the NF is $\sim 9 \mathrm{~dB}$. Moreover, a BER below $10^{-10}$ is achieved for all signal modes at $400 \mathrm{~Gb} / \mathrm{s}$. BER below $10^{-10}$ for $\mathrm{LP}_{01}$ signal mode is demonstrated for the different number of modes up to 12-spatial modes $(480 \mathrm{~Gb} / \mathrm{s}$ aggregate rate). The amplifier performance could further be improved by optimizing the core dopant distribution and by reducing the core-to-clad area ratio. We consider this an important step in extending SDM transmission to larger channel numbers with cost-effective and efficient amplification.

\section{REFERENCES}

[1] D. J. Richardson et al., Nature Photon. 7, 354 (2013).

[2] A. Li et al., J. Lightwave Technol. 30, 3953 (2012).

[3] P. Genevaux et al., Proc. OFC, Tu3C.5 (2015).

[4] Y. Jung et al., IEEE Photon. Technol. Lett. 26, 11 (2014).

[5] H. A. Youssef et al., Proc. ACP, ATh3A.81 (2014).

[6] Y. Jung et al., Opt. Exp. 22, 29008 (2014). 